\title{
Proceeding
}

Supplementary Issue: Summer Conferences of Sports Science. Costa Blanca Sports Science Events, 20-21 September 2019.

Alicante, Spain.

\section{Strength improvement in adults healthy men}

\author{
FRANCESCO PERROTTA ${ }^{1}$, ROBERTA ROSA ${ }^{2}$, GIUSEPPE MADONNA ${ }^{3}$ \\ ${ }^{1}$ M.I.U.R. Campania, Italy \\ 2IUL Telematic University, Italy \\ ${ }^{3}$ Parthenope University, Naples, Italy
}

\begin{abstract}
A strength is a physical vector quantity that manifests itself in the mutual interaction of two or more bodies, both at the macroscopic level and at the level of elementary particles. Quantifies the phenomenon of induction of a change in the state of rest or motion of the bodies themselves; in the presence of more strengths, it is the result of their vector composition that determines the variation of motion. The strength is classically described by Newton's second law as a temporal derivative of the momentum of a body with respect to time. A strength is often described as a push or pull. The strengths may be due to phenomena such as gravity, magnetism, or any other phenomenon that causes a body to accelerate. Keywords: Strength; Men; Strength improvement.
\end{abstract}

\section{Cite this article as:}

Perrotta, F., Rosa, R., \& Madonna, G. (2019). Strength improvement in adults healthy men. Journal of Human Sport and Exercise, 14(5proc), S1969-S1976. doi:https://doi.org/10.14198//hse.2019.14.Proc5.17

Corresponding author. M.I.U.R. Campania, Italy.

E-mail: giuseppe.madonna1984@gmail.com

Supplementary Issue: Summer Conferences of Sports Science. Costa Blanca Sports Science Events, 20-21 September 2019. Alicante, Spain.

JOURNAL OF HUMAN SPORT \& EXERCISE ISSN 1988-5202

(c) Faculty of Education. University of Alicante doi:10.14198/jhse.2019.14.Proc5.17 


\section{PURPOSE OF STUDY}

Highlight a broader picture of the strength expressed by the skeletal muscle system in the fit and healthy human being.

Later we will see how the mechanical strength is necessary for man to perform the functions of every day, from the simplest to the most complex, we also deepen the role of the levers to better understand the reasons and causes of our movements like a Figure 1 representation.

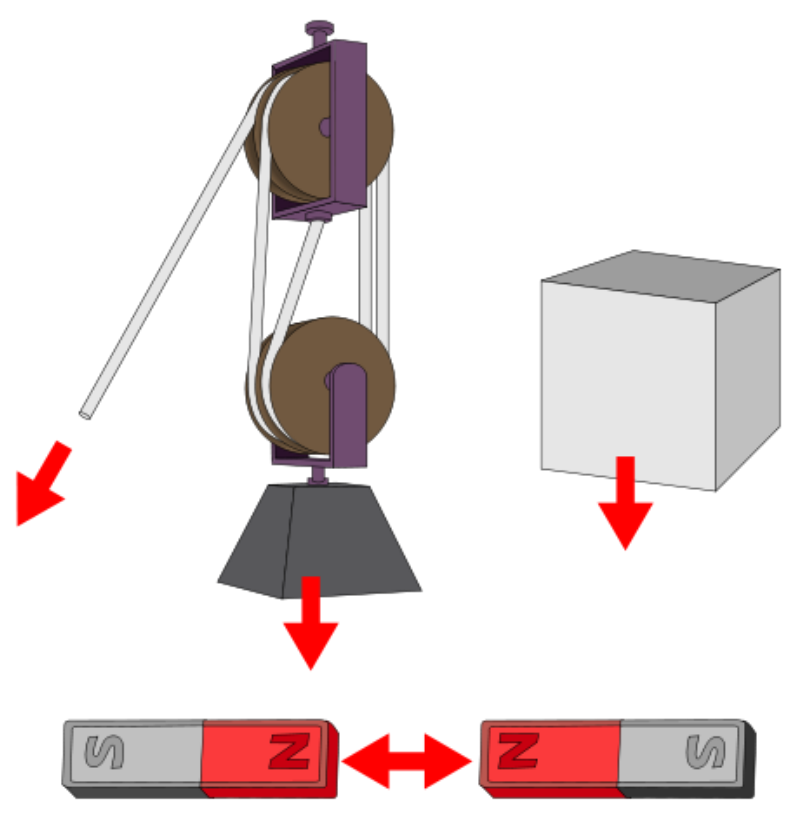

Figure 1. A strength is often described as a push or pull. The strengths may be due to phenomena such as gravity, magnetism, or any other phenomenon that causes a body to accelerate.

\section{RECENT FINDINGS}

There are three conditional capabilities:

- Resistance.

- Strength.

- Speed.

The development of these abilities counteract sarcopenia in adult men and benefits the person's posture and contrasts and prevents injuries.

Intramuscular coordination is useful for endurance athletes, who benefit from alternating fibre recruitment or even desynchronization, thus allowing greater recovery to those fibres that do not contract the multifrequency to create a base of neuro-metabolic strength and response with the multifrequency training and then bring all the fruits with the monofrequency at home. The latter needs the best techniques of exercise intensification (stripping, super-series, xrep, etc.), techniques that include in the medium term a stallion which, in the brief damage, seems to me hypertrophic and prevent the general syndrome to adapt to the maximum of supercompensation. 
We then have to Harre the subdivision of the strength in:

- Maximum strength: it is the highest strength that the neuro-muscular system is able to express with a voluntary contraction.

- Quick strength: it is the capacity of the neuro-muscular system to overcome resistances with a high rate of contraction.

- Resistant strength: it is the body's ability to oppose fatigue during strength performance over time.

The various expressions of strength mentioned above: maximum strength, explosive strength, resistance to explosive strength and muscular resistance can be classified according to biological principles. These expressions can be classified taking into account both the neuromuscular aspects, which serve to modulate the tension, and the metabolic aspects that determine its duration. Therefore, the maximum strength and the explosive strength are characterized by neurogenic factors, while the resistance to explosive strength and muscular resistance are characterized by metabolic factors.

Strength, speed, resistance is the sine qua non conditions for successful performance. The dominant capacity is that conditional capacity to which a motor performance requires a higher contribution. Most of the motor activities require an optimal performance of at least two qualities of the three listed. The development of one of the three conditional capacities must take place in a methodical way, since it directly or indirectly affects the others.

Strength is a fundamental ability that must be trained along with the others so as not to become counterproductive. The strength is in an athlete what the foundations represent for a home. Strength is the base from which to start. A cyclist cannot think of winning a final sprint if he has not trained strength, a volleyball player cannot think of jumping higher if he has not trained strength and a bodybuilder cannot think of developing further hypertrophy if he has not trained strength (Borde, Hortobágyi \& Granacher, 2015; Lesinski et al, 2015).

Of the three types of strength classified by Harre the maximum strength is the first to train. Once this quality has improved, then it can be transformed into other types of strength with adequate training. The maximal strength can then become, explosive, resistant or it can turn into hypertrophy.

The improvement of the maximum strength occurs first with adaptations and modifications at the level of the nervous system and then with morphological transformations that will lead to hypertrophy (Gebel et al, 2018; Hrysomallis, 2011; Kiers et al, 2013; Kibele et al, 2015).

Most likely, the neural adaptations act both at the central and the peripheral level, this determines as a final result a maximal activation of all the muscular fibres. These modifications will give the possibility to immediately recruit a very high number of muscles fibres, triggering all those processes that determine the explosive strength (Behm et al, 2010; Yamamoto et al, 2016).

The changes at the level of the nervous system will ensure that both the intramuscular and the intermuscular coordination improve, with consequent energy savings and increase in the speed of execution of a movement.

A fundamental concept, because it will be the means by which strength will be developed, is the concept of load. Small loads can get high tensions through the speed of execution of the movement but it is not optimal 
to use low loads and high repetitions because in this training situation the alternation of the recruitment of the motor units comes into play that does not lead to the improvement of strength. An elevated load will also give greater supercompensation. If no optimal muscle tension is produced through optimal loads, no increase in strength is produced (Behm et al, 2010).

The training methods to increase the maximum strength are varied and among these we remember:

Method of repeated efforts:

- Series method.

- Pyramidal method.

- Dynamic method.

- Maximal effort method.

- Static or isometric stress method.

- Contrasting methods.

The above methods are the result of studies by the authors mentioned at the beginning of the article and are methods that combine well with performance sports. Regarding the strength training for the body builder or the advanced fitness practitioner, the aim is to increase the recruitment capacity of the motor units, so that they can then be exploited in mesocycles for hypertrophic purposes.

In principle these are the characteristics to build the strength (in Figure 2)

\begin{tabular}{|r|rrrrrrrrrr}
\hline 1 & 1 & 2 & 3 & 4 & 5 & 6 & 7 & 8 & 9 & 10 \\
\cline { 2 - 12 } & $100 \%$ & $96 \%$ & $82 \%$ & $89 \%$ & $86 \%$ & $84 \%$ & $81 \%$ & $79 \%$ & $76 \%$ & $74 \%$ \\
9.5 & $98 \%$ & $94 \%$ & $91 \%$ & $88 \%$ & $85 \%$ & $82 \%$ & $80 \%$ & $77 \%$ & $75 \%$ & $72 \%$ \\
9 & $96 \%$ & $92 \%$ & $89 \%$ & $86 \%$ & $84 \%$ & $81 \%$ & $79 \%$ & $76 \%$ & $74 \%$ & $71 \%$ \\
8.5 & $94 \%$ & $91 \%$ & $88 \%$ & $85 \%$ & $82 \%$ & $80 \%$ & $77 \%$ & $75 \%$ & $72 \%$ & $69 \%$ \\
8 & $92 \%$ & $89 \%$ & $86 \%$ & $84 \%$ & $81 \%$ & $79 \%$ & $76 \%$ & $74 \%$ & $71 \%$ & $68 \%$ \\
7.5 & $91 \%$ & $88 \%$ & $85 \%$ & $82 \%$ & $80 \%$ & $77 \%$ & $75 \%$ & $72 \%$ & $69 \%$ & $67 \%$ \\
7 & $89 \%$ & $86 \%$ & $84 \%$ & $81 \%$ & $79 \%$ & $76 \%$ & $74 \%$ & $71 \%$ & $68 \%$ & $65 \%$ \\
6.5 & $88 \%$ & $85 \%$ & $82 \%$ & $80 \%$ & $77 \%$ & $75 \%$ & $72 \%$ & $69 \%$ & $67 \%$ & $64 \%$ \\
\hline
\end{tabular}

Figure 2. An RPE of 10 indicates that the assigned repetitions should be made without leaving any repetition aside, then zero buffer (margin). Look at the table. One repetition with RPE 10 is the ceiling, 100\%. Three repetitions with RPE 10 correspond to $92 \%$, which is therefore the maximum load with which you can do 3 repetitions, five repetitions at RPE 10 are $86 \%$ and so on. Up to here nothing difficult. In practice, if we read the table under the RPE 10 optics we are reading the classic table weight / repetitions possible.

\section{LEVER}

A lever is a simple machine, a device built by man to win by means of a strength, called the engine, another strength called resistant. The purpose of the primordial levers was to amplify the human strength allowing to carry out work not allowed by the simple use of muscle strength.

The levers obey a fairly simple physical principle: the system is in equilibrium if the resultant of the moments of the two strengths is null. 
In physics, the moment of a strength with respect to a centre is expressed by a module vector equal to the product of the intensity of the strength by the length of its arm (distance of the straight line of strength from the fulcrum).

\section{First type lever}

A lever is of the first type or of first kind if the fulcrum is between the driving strength and the resistant strength. In turn, the lever of the first type can be advantageous if the driving strength is more distant from the fulcrum of the resistant strength or, in the opposite case, disadvantageous. First type lever examples are: crowbar, scissors, tongs, etc.

\section{Second type lever}

A lever is said to be of second type or second kind if the fulcrum is on the same side of the driving strength and of the resistant strength, at the same time it is necessary that the driving strength is more distant from the fulcrum than the resistant. It is therefore deduced that the second type levers are always advantageous. They are second type levers, the wheelbarrow, the nutcracker, the bottle opener.

\section{Third type lever}

A lever is of a third type or of a third kind if the fulcrum is on the same side of the driving strength and of the resistant strength, at the same time it is necessary that the driving strength is closer to the fulcrum than the resistant. It is therefore deduced that the third type levers are always disadvantageous.

Also, the muscles of our body, inserted on the bones, are from the physical point of view of the levers. The figure shows the flexion of the forearm by the biceps brachii muscle.

This is a classic example of a third type lever, which as we have said is always disadvantageous. As the lever arm is shorter, the strength developed by the biceps muscle must be much greater than the weight strength of the ball held on the hand. This type of lever, however, allows a great amplitude and rapidity of movement.

In physics we speak of a static advantage and a dynamic advantage. There is a static advantage, when using a lower driving strength, you can win a greater resistance (advantageous lever), but in this case the speed and the amplitude of the movement are small, so there is a dynamic disadvantage.

Automatically a static disadvantage (disadvantageous lever) allows a greater speed and amplitude of movement, that is a dynamic advantage.

Static advantage $=$ dynamic disadvantage

Static disadvantage $=$ dynamic advantage

Plantar flexion of the foot with extended lower limbs is an example of a second kind of lever.

Resistance (weight) and strength (muscle) lie on the same side with respect to the fulcrum, the driving strength is farther and therefore the lever is advantageous. The very backward insertion of the sural triceps thus facilitates movement. 
The whole locomotor system is based on a levers system. Therefore, whenever there is movement, a lever is produced that can be of first, second or third type. In the human body the most frequent levers are those of the first and third type, while the advantageous third-type levers are very rare.

However, the human body uses some elements that facilitate the action of the muscles. This is the case, for example, of the patella which, by moving the traction strength of the quadriceps forward, increases its effectiveness.

\section{MAXIMUM STRENGTH}

Important factor to consider is the possible presence of hypertension that can seriously represent an impediment for such training, since loads very close to the ceilings determine an increase in blood pressure due to the Valsalva manoeuvre (exhalation to closed glottis), which inevitably occurs when it works with almost maximal loads for low repetitions; this leads to an increase in chest pressure and a reduction in the flow of venous blood to the heart. This condition is established especially during exercises involving large muscle masses such as the squat. This happens because it tends, unconsciously, to increase the intraabdominal pressure in order to protect the vertebral column from the stress.

For the athlete who is periodically subjected to medical checks or to everyone here who are constantly monitored, it is of crucial importance to cycle and periodize the workouts, always introducing a less long rest period before the maximum strength cycle or in any case a short break immediately after.

A question too often brought up concerns the number of series and repetitions optimal in the cycle dedicated to maximum strength. Several studies agree that there is no substantial difference between the 3 or 5 series per exercise, it is important to perform a low number of repetitions, not more than 4-7 strokes, with double and single.

The predominantly eccentric training, like that of the negative ones, represents a very powerful means for the development of maximum strength. With this training it is possible to brake loads of even $120-130 \%$ of 1RM. It should not, however, be followed for more than 2 or 3 weeks, so as not to overload the connective structures excessively.

Plyometric training can also be useful for increasing maximum strength, provided that the same rules are observed for negative repetitions. Both of these methods overload both the musculoskeletal system and the central nervous system.

The increase in strength is not exponential, its growth is therefore not always linear over time. If this were not the case in a few years any power athlete would be able to practice biceps curls with dumbbells of $200 \mathrm{~kg}$. Unfortunately, it is not like that. In strength training we must intervene gradually and in any case within human limits, setting realistic long-term goals that are therefore achievable.

\section{BENEFITS OF STRENGHT EXERCISE}

Stalemates or even regression of strength can be experienced which, however, must not give rise to worries or frustrations, but must motivate or suggest a period of rest from training. 
Many powerlifters will certainly find this topic familiar, not surprisingly these athletes always represent the "strongest" sports class, with a greater ability to recruit motor units under the voluntary control of the athletes of other disciplines.

Maximum strength is an extremely important conditioning ability and not training it in the right way is one of the biggest mistakes an athlete can make. If you want to achieve a decidedly high level of muscle growth and create an impressive physical structure, then you need to push hard and lift important loads.

Strengthens muscles and joints. With improvements also in posture and resistance to daily fatigue. Then, the abdominal and back muscles in the form distance the risk of pathologies of the vertebral column: hernias and lumbosciatalgie.

Optimize the metabolism. Improves the relationship between fat mass and lean body mass, the stimulus of hunger is regulated and the haematochemical parameters are rebalanced.

It increases the capacity and elasticity of the respiratory system. Improves the contractile capacity of the Heart and its coronary spraying: at rest, a sportsman has a lower heart rate than a sedentary person, and is less prone to sudden changes in pressure; moreover, its circulatory system is more elastic and has a better venous return, due to the greater efficiency of the musculature. Combats mood disorders. Physical activity is also good for the psyche and helps fight disorders such as anxiety and depression. Physical activity, in fact, contributes to the release of two important types of neuromediators: acetylcholine and endorphins. These are the molecules that produce the sensations of analgesia and well-being, properties that have led to the definition of the hormones of happiness.

\section{CONCLUSIONS}

The dominant capacity is that conditional capacity to which a motor performance requires a higher contribution. Most of the motor activities require an optimal performance of at least two qualities of the three listed. The development of one of the three conditional capacities must take place in a methodical way, since it directly or indirectly affects the others.

Strength is a fundamental ability that must be trained along with the others so as not to become counterproductive. The strength is in an athlete what the foundations represent for a home. Strength is the base from which to start. A cyclist cannot think of winning a final sprint if he has not trained strength, a volleyball player cannot think of jumping higher if he has not trained strength and a bodybuilder cannot think of developing further hypertrophy if he has not trained strength.

\section{REFERENCES}

Behm, D. G., Drinkwater, E. J., Willardson, J. M., \& Cowley, P. M. (2010). Canadian Society for Exercise Physiology position stand: The use of instability to train the core in athletic and nonathletic conditioning. Applied Physiology, Nutrition, and Metabolism, 35(1), 109-112. https://doi.org/10.1139/h09-128

Borde, R., Hortobágyi, T., \& Granacher, U. (2015). Dose-response relationships of resistance training in healthy old adults: a systematic review and meta-analysis. Sports medicine, 45(12), 1693-1720. https://doi.org/10.1007/s40279-015-0385-9 
Gebel, A., Lesinski, M., Behm, D. G., \& Granacher, U. (2018). Effects and dose-response relationship of balance training on balance performance in youth: a systematic review and meta-analysis. Sports Medicine, 48(9), 2067-2089. https://doi.org/10.1007/s40279-018-0926-0

Hrysomallis, C. (2011). Balance ability and athletic performance. Sports medicine, 41(3), 221-232. https://doi.org/10.2165/11538560-000000000-00000

Kibele, A., Granacher, U., Muehlbauer, T., \& Behm, D. G. (2015). Stable, unstable and metastable states of equilibrium: Definitions and applications to human movement. Journal of sports science \& medicine, 14(4), 885.

Kiers, H., van Dieën, J., Dekkers, H., Wittink, H., \& Vanhees, L. (2013). A systematic review of the relationship between physical activities in sports or daily life and postural sway in upright stance. Sports medicine, 43(11), 1171-1189. https://doi.org/10.1007/s40279-013-0082-5

Lesinski, M., Hortobágyi, T., Muehlbauer, T., Gollhofer, A., \& Granacher, U. (2015). Effects of balance training on balance performance in healthy older adults: a systematic review and meta-analysis. Sports medicine, 45(12), 1721-1738. https://doi.org/10.1007/s40279-015-0375-y

Yamamoto, S., Hotta, K., Ota, E., Mori, R., \& Matsunaga, A. (2016). Effects of resistance training on muscle strength, exercise capacity, and mobility in middle-aged and elderly patients with coronary artery disease: A meta-analysis. Journal of Cardiology, 68(2), 125-134. https://doi.org/10.1016/i.jicc.2015.09.005

\section{(9) $(\Theta \Theta \Theta$}

This work is licensed under a Attribution-NonCommercial-NoDerivatives 4.0 International (CC BY-NC-ND 4.0). 